Published in final edited form as:

Infant Behav Dev. 2015 May ; 39: 67-80. doi:10.1016/j.infbeh.2015.02.003.

\title{
Different assessment tasks produce different estimates of handedness stability during the eight to 14 month age period
}

\author{
Julie M. CampbelI ${ }^{1,2,{ }^{*}}$, Emily C. Marcinowski ${ }^{2}$, Jonathan Latta ${ }^{2}$, and George F. Michel ${ }^{1,2}$ \\ ${ }^{1}$ Center for Developmental Science, University of North Carolina at Chapel Hill, Chapel Hill, NC, \\ United States \\ 2Department of Psychology, University of North Carolina at Greensboro, Greensboro, NC, United \\ States
}

\section{Abstract}

Using 150 infants (57\% males), two common tasks for assessing infant hand-use preferences for acquiring objects were compared for their ability to detect stable preferences during the age period of eight to 14 months. One task assesses the preference using nine presentations of objects; the other uses 32 presentations. Monthly classifications of hand preference for each task were determined by either a commonly used a decision criterion in which one hand is used $50 \%$ more often than the other or a criterion based on proportion of hand-use difference that exceeds a conventional alpha probability of 0.05 . The seven monthly assessments provided by the two tasks also were examined for latent classes in their developmental trajectories. The two tasks were significantly different for both their identification of latent classes and their monthly classification of the infant's hand-use preference. The 32 presentations yielded three developmental trajectories (45\% right preferring, $5 \%$ left preferring, and 50\% no clear preference) whereas the nine presentations revealed only two trajectories (70\% right, $30 \%$ no preference). The nine presentations task, with the $50 \%$ proportion decision criterion, was very generous in classifying right and left-preferring infants at each month but produced greater fluctuations across months compared to the 32 presentation task with an alpha decision criterion. Both tasks revealed that a large proportion of infants are still developing a hand-use preference during this age period. Recommendations are made for examining the development of hand-use preferences and their relation to the development of other neuropsychological functions.

\section{Keywords}

Handedness; infancy; grasping; group-based trajectory models; latent classes

\footnotetext{
(C) 2015 Published by Elsevier Inc.

*Corresponding Author: University of North Carolina Greensboro, Department of Psychology, 296 Eberhart Building, Greensboro, NC, 27402, Phone: (336) 334-4046, Fax: (336) 334-5066, jmcampb2@uncg.edu.

Publisher's Disclaimer: This is a PDF file of an unedited manuscript that has been accepted for publication. As a service to our customers we are providing this early version of the manuscript. The manuscript will undergo copyediting, typesetting, and review of the resulting proof before it is published in its final citable form. Please note that during the production process errors may be discovered which could affect the content, and all legal disclaimers that apply to the journal pertain.
} 


\section{Introduction}

The primary goal of this study was to compare two common tasks for assessing infant handuse preferences for their ability to identify stable preferences. Many investigators have considered handedness to be an unstable trait during its early development with some proposing that handedness cannot be identified reliably until the ages of four to seven years (McManus et al., 1988) or as late as six to 10 years of age (Fennell, Satz, \& Morris, 1983; Gesell \& Ames, 1947). Consider this recent summary statement: "Infants initially use both hands indifferently (Corbetta \& Thelen, 1999; Rönnqvist \& Domellof, 2006), then preference for one hand becomes clear generally from 18 months of age on (Fagard \& Marks, 2000) and is more and more pronounced during the following years (Ingram, 1975)" (Dubois et al., 2009, p. 414). Thus, a general consensus has arisen that restricts reliable handedness assessment to the period beyond infancy (Corbetta \& Thelen, 1999, 2002; Fagard, 1998; Fagard \& Lockman, 2005; McCormick \& Maurer, 1988; Piek, Gasson, Barrett, \& Case, 2002; Thelen, 1995; Thelen, Corbetta, \& Spencer, 1996). Indeed, most longitudinal studies beginning during infancy have revealed much within-individual variability across age (Corbetta \& Thelen, 1999, 2002; Fagard, 1998; Ferre, Babik, \& Michel, 2010; Michel, Babik, Sheu, \& Campbell, 2014; Piek et al., 2002; Thelen, Corbetta, \& Spencer, 1996).

However, some evidence indicates that infant hand-use preferences for a skill such as the acquisition of objects are relatively stable across assessment periods for a majority of infants during the age range of seven to 13 months (Michel, Sheu, \& Brumley, 2002; Michel, Tyler, Ferre, \& Sheu, 2006). Using a reliable and valid procedure capable of identifying significant differences in the frequency of use of each hand at each monthly assessment during the 6- to 14-month period, Michel, Babik, Sheu, \& Campbell (2014) assessed the longitudinal character of the hand-use preference for acquiring objects for a large sample of 328 infants (182 males). This large sample size permitted the use of a group based trajectory model which revealed three potential latent classes in these nonlinear, somewhat fluctuating, individual developmental trajectories. The three groups represented those with a right handuse preference (38\%), those with a left hand-use preference (14\%) and those without a clear preference (48\%) but whose trajectories were significantly trending toward a right hand-use preference.

Thus, although the actual preference scores of hand-use varied across the months for the left-hand and right-hand preferring infants, the pattern was consistent enough to reveal distinct trajectories. These trajectories demonstrated that right-hand preferring and left-hand preferring infants increased and maintained their hand preference for acquiring objects across the six to14 month age period. Consequently, fluctuations in scores may indicate less about fluctuations in handedness development and more about the influence of transitory factors in a rapidly developing nervous system (cf., Babik, Campbell, \& Michel, 2014). Only the large sample size with frequent longitudinal assessments enabled the identification of the three groups as distinguished by the developmental trajectory of their hand-use preferences. 
Indeed, when infant hand-use preferences were classified for each month (across the six to 14 month age period) using a z-score decision criterion ( $\mathrm{p}<0.05)$, Michel et al. (2014) found that the 12 month assessment was the best at identifying left-handed and right-handed infants who were identified also by the latent class analysis as having the that same handedness (less than $1 \%$ of 328 infants had their latent class hand-use preference misclassified by the z-score decision criterion). Nevertheless, even at 12 months, nearly $40 \%$ are likely to be classified differently between the latent class and z-score decision criteria, with most shifting between the hand preference and no preference categories across the two techniques. Thus, a single assessment at any month during infancy is likely to miss the important trajectory aspects of infant handedness development and the nature of that error is likely to vary greatly according to the age at assessment. This raises important problems for assessment of infant hand-use preference, especially for cross-sectional designs.

If infant hand-use preference reflects the consequences of a markedly immature but rapidly developing nervous system, then it may be sensitive to differences in assessment procedures and conditions. It is likely that hand-use preferences may be described as unstable and variable during infancy because of variation across studies in sample sizes, longitudinal sampling rates, and the types of skills being assessed (e.g., swiping at objects, grasping and acquiring them, engaging in role-differentiated bimanual manipulation). Given the wide array of assessment methods and decision criteria used for classifying hand-use preferences, a meta-analysis on the development of infant hand-use preferences or the relation of these preferences to other infant or childhood functions would be misleading and inappropriate.

Since some of the variability in infant hand-use preferences likely derives, in part, from variability in the developmental succession of different kinds of manual skills within which a hand preference may be expressed, the type of manual skill assessed and timing of the assessment becomes critical. We propose that object acquisition skills should be the focus of studies designed to assess the trajectory of the development of hand-use preferences during the period from 6 to 14 months of age for the following reasons. Object acquisition is a prevalent (and sufficiently challenging) manual skill in the infant's repertoire during this period. Also, object acquisition develops after establishment of the skills of reaching for and contacting objects (Michel \& Harkins, 1986) and it is incorporated into all other manual skills involving object manipulation (e.g., tool use and artifact construction). Hand-use preference for acquiring objects is related to and predicts the later development (at about 11 to 12 months) of a hand-use preference for unimanual object manipulation (Hinojosa Sheu, \& Michel, 2003; Campbell, Babik, \& Michel, submitted) and a hand-use preference for role differentiated bimanual manipulation, which appears at about 13 to 14 months (Babik \& Michel, submitted). Similarly, Fagard and colleagues have used object grasping (similar to object acquisition) to characterize infant hand-use preferences during the first year and its relation to tool-using and gesturing (e.g., Esseily, Jacquet, \& Fagard, 2011; Fagard \& Marks, 2000; Jaquet, Esseily, Rider, \& Fagard, 2012; Sacco, Moutard, \& Fagard, 2006). Therefore, acquisition of objects seems pivotal for investigating the early development of hand-use preferences and will be the focus of this study.

Michel $(1991,2002)$ has argued that the criteria used to define a preference can affect its apparent stability (Michel, Sheu, \& Brumley, 2002). For example, calculating a hand-use 
preference using a laterality index (i.e., the relative difference between hands divided by the total frequency of hand use, Ramsay, 1980) and classifying the infant's preference with a subjective decision criterion (e.g., proportions greater than $+50 \%$ or less than $-50 \%$ identify right and left preferences, respectively) may show less stable preferences across assessment ages than a preference defined by statistical estimates of whether the inter-manual differences are likely to have occurred by chance (Michel et al., 2002; Michel, Tyler, Ferre, $\&$ Sheu, 2006). Although a laterality index exhibits a continuous distribution across individuals with a "right shift" skew similar to that observed in adult handedness (Annett, 1972), there is no common agreement about how to classify individuals into relatively discrete handedness groups.

For example, Esseily, Jacquet, and Fagard (2011), among others, have used a laterality index for grasping objects $(\mathrm{GLI}=(\mathrm{R}-\mathrm{L}) /$ total actions) with proportions greater than +0.5 or less than -0.5 designating, respectively, right and left hand-use preference. Infants with a GLI between these scores are considered to have no preference. Another technique for classifying infants based on their laterality index would be to use a z-score transformation of the index $\left(\mathrm{z}=(\mathrm{R}-\mathrm{L}) /(\mathrm{R}+\mathrm{L})^{1 / 2}\right)$ to estimate of the probability of obtaining the intermanual difference in frequency of use by chance $(\mathrm{z}>1.9=$ right hand bias and $\mathrm{z}<-1.9=$ left hand bias) at a Type I error rate of less than 0.05 (Michel et al., 2002). This would provide a "conventional" measure of confidence for the classification. However, the z-score transformation of the laterality index requires a frequency of left and right hand-use of at least 25 at each assessment and no instances in which both hands were used. Thus, one aim of the present study is to compare the consequences of using the a common laterality index (e.g., Esseily et al., 2011) and decision criterion (+/- 0.5) to that of using the a z-score transformation of the index (Michel et al., 2002) with a classification decision criterion (p < 0.05 ) on the appearance of stability of hand-use preferences during infancy.

Many studies use too few opportunities (items) for assessing the infant's hand-use preference to use a z-score transformation. For example, Sacco, Moutard, \& Fagard (2006) used only seven items (with two items presented twice to yield nine presentations to the infant) for each assessment of a hand-use preference. Too few presentations of items can create the appearance of instability across assessments and it can distort the identification of the infant's preference. For example, the binomial test of hand-use with nine presentations requires that the same hand be used for at least eight of the nine presentations for the preference to be significant at alpha $=0.05$, two tailed. In contrast, seven right hand uses of the nine presentations create a proportion of 0.55 , which would be labeled as a right preference.

Recently, Esseily et al. (2011) presented eight items to 22 infants who were 14 months of age. Their calculation of the GLI revealed that $64 \%$ of the infants were right-handed, $16 \%$ were left-handed, and 23\% had no preference. In contrast, Michel et al. (2014) using 31 items (one presented twice) presented to 328 infants at 14 months and a z-score alpha of 0.05 decision criterion found that $39 \%$ of infants were right-handed, $7 \%$ were left-handed and $54 \%$ had no preference. Clearly, differences in the decision criterion and/or the number of items in the assessment technique make a difference in identifying the infant's handedness at 14 months. Thus, another aim of the present study is to compare the use of a 
31 item assessment procedure (Michel, Ovrut, \& Harkins, 1985) with a seven item procedure (Sacco et al. 2006) for characterizing infant handedness. In both cases, a conventional alpha $=0.05$ criterion will be compared with that of the commonly used GLI proportions of $+/-0.5$.

Aim one of the current study compares two different infant hand-use preference assessment techniques (e.g., Esseily et al, 2011 vs. Michel et al., 2014) that measure the infant's ability to acquire objects at seven monthly assessments from eight to 14 months of age. The two assessment techniques differ in the number of item presentations used to identify a preference (nine versus 32) and they also differ in the decision criteria used for assigning infants into one of three handedness groups (left, right, or no preference). We predict significant differences between the decision criteria for classification using the eight-item assessment with the $+/-0.5$ classification criterion resulting in a much less conservative estimate of the infant's handedness. Also, we predict that eight-items will produce statistically different distributions of the infants into the three classes of handedness (right, left, no preference) compared to the 31 item assessment, regardless of the decision criteria used for classification.

Aim Two of the study compares the classification of infant hand-use preference derived from each of the two assessment techniques (nine presentations and 32 presentations) using a group based trajectory model of the seven monthly assessments. This trajectory analysis permits identification of individual and group level variability in hand-use preferences and the effect of the different assessment techniques on the appearance of instability in infant hand preference status, the character of its developmental trajectories, and the rate of its developmental change. We predict that the two techniques for assessing hand-use preference during infancy will yield differences in identified latent classes of hand-use preference. Also, these trajectory classifications will be compared with a classification that is derived from the assessment at each of the seven months in order to assess the differences in those hand-preferences identified by longitudinal data and those derived from data from a single age.

The main goal of this study is to facilitate the development of techniques for assessing infant hand-use preferences that will enable its reliable characterization so that the development of hand-use preferences can be examined in relation to the development of other important psychological functions during infancy (e.g., tool use, (Rat-Fischer, O'Regan, \& Fagard, 2012); gestural communication (Esseily et al., 2011; Jacquet et al., 2012); language ability (Nelson, Campbell, \& Michel, 2013)). If one method of assessing and classifying handedness resulted in a substantial proportion of infants classified differently from another method, then any results from studies using these different methods in relation to the development of other psychological functions must be treated with caution.

\section{Materials and methods}

\subsection{Participants}

Participants were 150 infants (64 or $43 \%$ females) recruited from birth records obtained from the Guilford County courthouse in Greensboro, North Carolina. All had full-term 
pregnancies and births without complications. Procedures for recruitment, obtaining informed consent and data collection were in accordance with the regulations set by the UNCG Institutional Review Board for the protection of human subjects. Our sample comprised 58\% Caucasian, 22\% African American, 4\% Hispanic, 2\% Asian, 1\% Pacific Islander, $9 \%$ multiracial infants, (4\% chose not to answer), which is roughly representative of Guilford County's ethnic demographics (US Census Bureau, 2010). For each visit, parents were given a $\$ 10$ Target gift card. At these visits, infants were administered two handedness assessments: the "common" laterality task (e.g., Esseily et al., 2011) and the "Michel" handedness task (e.g., Michel et al., 2014). The 150 infants were included in the data analyses only because they missed no more than one monthly visit. Twelve infants missed one visit due to illness or a schedule conflict. The missing visits occurred at the following months: two at eight months, three at nine months, one at 10 months, one at 11 months, two at 12 months, and three at 13 months. Ninety infants (60\% of the sample) were participants in a previous publication (Michel et al. 2014).

\subsection{Procedures and Materials}

Infants were brought to the Infant Development Center within seven days of their birth date from 8 to 14 months at monthly intervals ( 7 visits total). The eight month age was chosen because pilot data collection revealed that mothers of infants younger than eight months were quite concerned about a potential choking hazard for some of the items and IRB approval required shifting to the eight month age. The 14 month age was chosen as the oldest age for which the "Michel" laterality task has published data (Michel et al., 2013). The experimenter sat directly across from the infant on the convex side of a rounded crescent-shaped table, while the infant sat on the concave side. The infant sat on the parent's lap, so the infant's navel was at table height, leaving the arms unconstrained. The parent sat close to the table and held the infant on either side of the infant's waist to maintain a stable posture. A camera (Panasonic WV-CP240) was placed to the side and directly above the infant's hands, allowing two views for coding accuracy. Each visit was recorded in its entirety for later data coding. Both tasks were in the same setting. There were two hand-use preference tasks administered at the visit, the "Fagard" task (e.g., Esseily et al., 2011) and the "Michel" task (e.g., Michel et al., 2013) from eight to 14 months of age. For every infant, at each visit, the "Fagard" task was presented after the "Michel" task. Since the Michel task took less than 12 minutes to administer and the Fagard task took less than four minutes to administer, no infant was lost to fussiness during the sessions. Therefore, any differences between the tasks are unlikely to reflect differences in infant state.

Toys for the "Fagard" task were provided through the generosity of Dr. Jaqueline Fagard (see Appendix IB). The procedure also was demonstrated personally by Dr. Fagard in order to ensure proper replication (see Esseily et al., 2011 for more details of the procedure). In the "Fagard" task, nine trials of seven objects are given to elicit simple grasping ( 2 trials of one Playmobil® figure, 1 trial of another Playmobil ${ }^{\circledR}$ figure, a blue teether ring, and a shaker), precision grasping (red tube in a plastic jar, and a horse in a cup), and bimanual manipulation ( 2 trials of a plastic tube in a long, wooden container). The Playmobil@ figures and the shaker were presented to the infant's midline on the table. The red tube in a plastic jar and the horse in a cup were presented to the infant's midline, and their containers (jar/ 
cup) were held on the table by the presenter. The blue teether ring was presented to the infant's midline in the air at chin-level. Finally, the bimanual manipulation toys were shown to the infant's midline at chin-level, and placed into the infant's reaching hand. The hand used to demonstrate each toy was varied in a pseudo-random fashion. It must be noted that the "Fagard" task was chosen only because it is among the most commonly used assessment techniques for measuring infant hand-use preferences and shares many characteristics with other techniques (e.g., a relatively small number of presentations, the $+/-0.5$ proportion for classifying a preference).

In the "Michel" task, 32 trials of 31 objects of varying shapes and sizes were given to the infants separately from ages eight to 14 months (see Appendix IA). These objects were meant to interest the infant enough that they would be more likely to pick up and manipulate them. The objects were presented either singly (22) or in pairs (10). Single objects were presented either on the table (28) or in the air (3) to the infant's midline, in line with the infant's nose (see Babik et al., 2014 for more details). Air objects were held $20 \mathrm{~cm}$ from the infant's shoulders and $12-15 \mathrm{~cm}$ above the table. The paired objects were two identical objects placed on the table in line with the infant's shoulders. All paired objects were presented on the table. The presenter allowed the infants to manipulate each object until it had been picked up or after 20 seconds had elapsed (whichever occurred first). The hand(s) initially used to pick-up the object(s) were recorded by the camera for later coding. Moreover, "tree-based" non-linear regression methods with 153 infants (75 males) assessed every other month from 7 to 13 months of age demonstrated that the toys used in this assessment technique are unbiased stimulus items (they do not affect the hand-preference) regardless of the age or sex of the infant (Baker, 1998).

\subsection{Data Coding}

The software program Noldus (-) Observer XT 10.1 was used to code the videos in both tasks. This program allows coders to stop or slow down the videos, and mark the precise millisecond of an action. Each object presentation was initially watched in real time. When relevant behaviors were observed, the video was then slowed down to mark the action. Once the presentation's actions were marked, the coder re-watched the entire presentation to ensure accuracy. When coding the "Michel" task, coders would mark which hand(s) initially picked up a toy or if the infant did not manipulate the object after 20 seconds that object was marked as such. When coding the "Fagard" task, coders would mark which hand(s) a) initially acquired the simple grasp toys, b) initially acquired the objects from the containers for the precision grasp toys, and c) initially pulled the plastic tube out of the wooden container for the bimanual manipulation toy. Only bimanual pulls were counted when coding the bimanual manipulation toy, and any other action was not counted towards the total. Again, Dr. Fagard generously advised us on how to code hand-use for these toys including the coding for the use of "both" hands (personal communication, June 25, 2010).

Both inter-rater and intra-rater reliabilities were conducted on $20 \%$ of randomly selected videos. The "Michel" task had an inter-rater reliability of $90.5 \%$ agreement and an intrarater reliability of $96.9 \%$ agreement. The "Fagard" task had an inter-rater reliability of $91.45 \%$ agreement and an intra-rater reliability of $96.63 \%$ agreement. 


\subsection{Determination of Handedness Groups}

For both the "Fagard" and "Michel" tasks, an infant's hand-use preference was calculated in two ways: First the Grasping Laterality Index (GLI): (Right-hand grasps-left hand grasps) divided by all grasps, including bimanual ("both") grasps was calculated; Second, the GLI was calculated but with the bimanual grasps eliminated from the denominator. This latter calculation would emphasize lateralized differences in hand-use. For both sets of calculations and for both tasks, infant hand-use preferences were identified in two ways: First, infants were classified as right-hand preferring if their GLI score was $\geq+0.5$, left-hand preferring if their GLI score was $\leq-0.5$, or as having no preference if their GLI score fell between 0.5 and -0.5 . Second, an infant's hand-use preference was determined using the binomial test so as to identify significant (alpha $\leq 0.05$ ) differences between the hands in their frequency of use. Thus, if the probability for the number of grasps was less than alpha $=0.05$, then an infant was considered to have a preference for the hand performing the greater number of grasps (right or left). If $\mathrm{p}>0.05$, then the infant was classified as demonstrating no reliable hand-use preference (i.e., "no preference").

The binomial test was used in place of the published z-score transformation of the laterality index (Michel et al., 2013) for two reasons. First, the "Fagard" nine presentation task could not be transduced reliably to a $\mathrm{z}$-score. Second, the 150 infants in the current study generated a much larger number of bimanual object acquisitions ("boths") than had been observed previously. When numbers of lateralized responses become less than 25 , the zscore transformation cannot reliably represent the probabilities of differential frequency of hand use. Therefore, the binomial test (with alpha $=0.05$ indicating significant differences between the hands) was used as the criterion for classifying infant hand-use preferences for both tasks. With numbers of lateralized responses greater than 25 , the z-score transformation approximates the binomial distribution (Siegel, 1956). Since there were seven monthly assessments of each infant's hand-use preference during the eight to 14 month period, each of the two assessment tasks could be examined and compared for latent classes in the developmental trajectories of infant handedness.

\section{Results}

\subsection{Comparison of assessments using Chi-Square analyses}

In order to determine whether our conduct of the "Fagard" procedure was equivalent to that of published results from her lab, we compared our results with those of the Esseily et al. (2011) study (Table 1). The percentages in each handedness group were derived by using the GLI with the decision criterion of greater than +0.5 or less than -0.5 to determine handedness status for infants at 14 months of age. A chi-square analysis revealed that the distribution of infants in each handedness group is not significantly different, $\mathrm{X}^{2}(2)=3.88$, $\mathrm{n}=162, \mathrm{p}=.14$. Thus, using the same task and decision criterion, we have replicated Esseily et al.'s (2011) study of infant handedness at 14 months of age. Thus, we believe that our conduct of the "Fagard" task is comparable to that of Fagard and her colleagues.

Aim One of this study was to compare the two methods of assessing infant hand-use preferences (the "Fagard" nine presentations method vs. the "Michel" 32 presentations 
method) at each month from eight to 14 months. Aim one also permitted evaluation of the two decision criteria used to classify the infant's hand-use preference: "Fagard's" proportion criterion verses a conventional statistical decision criterion (a binomial test with an alpha of $<0.05$ specifying a difference in use between the two hands that was unlikely to occur by chance). Chi-square analyses were used to determine whether the number of infants classified into each handedness group differed according to the classification criteria at each month (chi-square alpha values were Bonferoni corrected for multiple comparisons to $\mathrm{p}=$. 007). Figure 1 shows the percentages of infants in each hand-use preference category at each month for the "Fagard" nine presentations task with Figure 1A showing the percentages with the binomial decision criterion and Figure 1B showing the percentages with the proportion criterion. The chi-square revealed a significant difference between the two decision criteria for classifying hand-use preferences at each of the seven ages (see Appendix IIA).

Note that the binomial decision criterion reduces the proportion of infants with a hand-use preference and identifies most as having no preference when compared to the proportional decision criterion for identifying the infant's hand-use preference (Figure 1A compared to Figure 1B). Both of these decision criteria included bimanual acquisitions in the calculation (as does the published work of Fagard). Thus, Figure 1 shows that the nine presentations with a proportion decision criterion for specifying a hand-use preference over-estimates the likelihood that an infant exhibits a hand-use preference. By over estimating the presence of a preference, this technique likely would reveal changes in hand-use preference status between assessment ages and generate the appearance of developmental instability in the infant's hand-use preference. The use of a binomial decision criterion with only nine presentations results in the overwhelming majority of infants without a hand-use preference. Thus, nine presentations may be inadequate to assess the infant's hand-use preference.

Figure 2 shows the percentages of infants in each handedness class at each month for the "Michel" 32 presentations method according to the two decision criteria. Chi-square analyses (Bonferroni corrected to $p=0.007$ ) revealed that the distributions are significantly different only for 14 month age between the two decision criteria for classifying hand-use preferences (Appendix IIB). With 32 items and a $+/-0.5$ proportion decision criterion (Figure 2B), the overwhelming majority of infants (77 to 90\%) exhibit no hand-use preference for each of the seven monthly assessments. In contrast, using a binomial decision criterion, 69 to $75 \%$ of infants exhibit no hand-use preference (Figure 2A). Thus, Figure 2 shows that the 32 presentations with a proportion decision criterion for specifying a handuse preference under-estimates the likelihood that an infant exhibits a hand-use preference. By under estimating the presence of a preference, this decision criterion likely would reveal changes in hand-use preference status between assessment ages and generate the appearance of developmental instability in the infant's hand-use preference. In contrast, using a binomial decision criterion for classifying the infant's hand-use preference (Figure 2A), it is likely that there will be greater consistency of hand-use preference within infants across assessment ages which would contribute to the appearance of developmental stability of handedness for many infants. Again, these analyses include bimanual acquisitions in the calculation of the proportions and the binomial which reduces the likelihood of obtaining a lateralized difference. 
Figure 3 compares the handedness distribution for each month for the "Fagard" nine presentation method (Figure 3A) and the "Michel" 32 presentations method (Figure 3B) using the proportion decision criterion. Appendix IIC shows the results of the chi-square analysis of the difference between the two tasks (the distributions are significantly different at all ages but 11 months). In both methods, the laterality index was calculated without the inclusion of bimanual acquisitions so as to emphasize lateral differences between the hands. Note that the nine presentations identifies that the majority of infants have a hand-use preference during the last three months (Figure 3A). In contrast, the 32 presentations method reveals that a majority of infants at each age do not exhibit a hand-use preference at any age. This suggests that the proportional decision criterion may be too liberal in identifying a hand-use preference during this age range.

Figure 4 compares the proportion of infants in each handedness category at each age for the "Fagard" task and proportion decision criterion (Figure 4A) with the "Michel" task and binomial decision criterion (Figure 4B), without the inclusion of bimanual acquisitions. Appendix IID shows there were no significant differences in distributions of hand-use preference at each month between the two tasks using a chi-square analysis. Removing the bimanual manipulations from the analysis shows a relatively stable proportion of right- and left-hand preferring infants for the "Michel" task and decision criterion but about 50\% of the infants do not exhibit a hand-use preference at each age (Figure 4A). In contrast, the "Fagard" task and decision criterion reveals that a majority of infants exhibit a hand use preference by 14 months (Figure 4B). Comparing Figure 4A with Figure 1B (Appendix IIE), we can see that the "Michel" task and decision criterion identifies a different proportion of infants in each hand-use preference category at each age than does the "Fagard" task and decision criterion. Employing either of these two tasks will yield fundamentally different numbers of infants with a hand-use preference at any of these seven ages. Moreover, after their first year (at 13 and 14 months), the "Fagard" task and decision criterion reveals that a majority of infants manifest a hand-use preference (right- and left-preference) in contrast to the "Michel" task and decision criterion which reveals that $50 \%$ or more of the infants do not manifest a statistically reliable hand-use preference at any age.

\subsection{Comparison of assessments using group based trajectory models}

Aim Two of the study was to compare the classification of infant hand-use preferences using a group based trajectory model derived from the seven monthly assessments using either the "Fagard" task or the "Michel" task. To address this aim, two longitudinal analyses of hand-use preference scores were conducted using a group-based trajectory model (GBTM) for each task. Group-based trajectory modeling (GBTM) clusters similar patterns of trajectories together and identifies subgroups within a sample. The infants within each identified sub-group tend to follow a similar developmental trend BUT differ from infants within other sub-groups across the entire sample (it's a similar idea to latent class analysis, but for trajectories instead of single data points). The SAS PROC TRAJ procedure and the Bayesian information criterion (BIC) were used to identify the number of latent hand-use preference groups present in the data (Jones, Nagin, \& Roeder, 2001; Schwarz, 1978). Initially, quadratic models were tested based on Michel et al.'s (2013) findings (derived from nine monthly assessments from 6 to 14 months of age for 328 infants) that infant hand- 
use preference trajectories are quadratic. Since the seven monthly assessments for the present study did not reveal any significant quadratic trends for any model, all models listed below are linear.

Figure 5 shows the two latent class hand-use preference groups that were revealed in the "Fagard" task. A "right hand-use preference" group comprised $70 \%$ of the sample; it began with a significant right hand-use preference and increased that preference with age ( $\beta_{0}=0.20$, $\left.p<0.01 ; \beta_{1}=0.05, p<0.01\right)$. The second latent class that was revealed appeared to be a "no preference" group (30\% of the sample) because the origin did not differ from zero and there was no significant slope across age $\left(\beta_{0}=-0.08, p=0.17 ; \beta_{1}=-0.01, p=0.67\right)$.

The GBTM analysis of the "Michel" task revealed three groups (Figure 6). A "right handuse preference" group ( $45 \%$ of the sample) started at eight months with a significant right preference and retained it $\left(\beta_{0}=0.31, p<0.00 ; \beta_{1}=-0.00, p=0.99\right)$. A second group ( $50 \%$ of the sample) are labeled "no preference" because their intercept and slope showed no difference from zero ( $\beta_{0}=0.01, p=0.74 ; \beta_{1}=0.01 ; p=0.12$ ). A third group ( $5 \%$ of the sample) began at eight months with a significant left hand-use preference and remained left $\left(\beta_{0}=-0.30\right.$, $\left.p<0.001 ; \beta_{1}=-0.01, p=0.71\right)$. Thus, the "Michel" task revealed three groups representing those infants whose hand-use preference trajectories could be characterized best as no preference, right-, and left-preference.

Table 2 demonstrates the concordance of hand preference assignment between the latent class analysis for the "Michel" task and that derived from the binomial test at each age. The Kappa tests show a "fair" strength of agreement between the two classification techniques with one percent or less discordance of "lateralized" preference at each age. Thus, the 32 presentations at each month and a binomial decision criterion capture the hand-use preferences revealed by the latent class trajectory analysis fairly well. In contrast, the Kappa tests reveal a "poor" strength of agreement between the latent class analysis for the "Fagard" task and the proportion decision criterion at each age. Using nine presentations (seven items) at each month does not capture the type of hand-use preference captured by the latent class trajectory analysis derived from 32 presentations at each month.

\section{Discussion}

Aim one of the current study was to compare two common assessment tasks that measure the infant's hand-use preference to acquire objects at seven monthly assessments from eight to 14 months of age. The number of presentations for assessing the infant's hand use preference and the decision criterion for classifying the preferences resulted in significant difference in the distribution of infants among the three hand-use preference groups (rightpreferring, left-preferring and no preference). The "Fagard" task, nine presentations with a proportion decision criterion, overestimated the number of infants with a hand-use preference at each age when compared with a binomial decision criterion. The "Michel" task, 32 presentations, seemed to be much more robust for the two decision criteria. Only one age period (14 months) revealed a significant difference in the distribution of hand-use preference classification according to the proportion versus the binomial decision criterion for the "Michel" task. Moreover, using just the proportion decision criterion, the "Fagard" 
and "Michel" tasks were significantly different in the distribution of infants among the handuse preference categories for each age period except 11 months. The "Fagard" task identified many more infants as having a hand-use preference than did the "Michel" task. Therefore, when using a proportion decision criterion, the "Michel" task is more conservative in assigning a preference than is the "Fagard" task. This difference would likely result in the identification different relationships between infant hand-use preferences and performance on other measures of infant cognitive and sensorimotor functions.

When bimanual acquisitions were removed from the analyses of hand-use preference, there were no significant differences in the distribution of infants between the "Michel" task (using a binomial decision criterion) and the "Fagard" task (using a proportion decision criterion). However, this is likely the result of both tasks identifying an overwhelming majority of infants has having no preference at all ages. When the original procedure of the "Michel" task (with a binomial decision criterion and without including bimanual acquisitions) is compared with the original procedure of the "Fagard" task (with a proportion decision criterion and including bimanual acquisitions), these two tasks are statistically different in their estimation of the infant's hand use classification at each age. Thus, a handuse preference assessment procedure which employs nine presentations and uses the proportion of the laterality index to classify infants into different hand-use preference categories provides a different account of infant hand-use preferences than does an assessment which has 32 presentations and uses the statistical procedure (binomial formula with an alpha level of 0.05) to classify infants. The fact that these assessments classify infants into different hand-use preference categories has implications for the feasibility of studying infant hand-use preferences reliably and for drawing conclusions about the character of handedness development during infancy. Whereas one method identifies more lateralized individuals, the other identifies them as having "no preference", contributing to confusion in the literature.

What cannot be concluded from the current study is whether it is the type of objects used or the number of assessment trials that is affecting the differences observed between the two common procedures for assessing infant hand-use preferences. Would two or three presentations of the same objects in the "Fagard" task yield results more comparable to the "Michel" task? If so, then this would indicate that it is the number of presentations and not the object types that generates the differences between the tasks. Given that there are some similarities among the objects of the two tasks and that there are the differences in the decision criteria for forming groups, we suspect that it is unlikely to be simply the objects that are creating the differences observed. However, that question awaits specific investigation.

The second aim of this study was to compare the two assessment tasks for their ability to track trajectories in the development of hand-use preferences. Although there may be variations across age in the score of an infant's hand-use preference, it is possible to examine the trajectories for latent classes. The "Fagard" task identified only two latent classes with a large majority of infants exhibiting a right hand-use preference (70\%) and a minority exhibiting no preference. No left preferring infants were identified in the trajectory analysis of the "Fagard" task despite the ability of this task to specify left-preferring infants 
at each age. In contrast, the "Michel" task identified three latent classes in the trajectories of hand-use development during this age range, with $50 \%$ of the infants having no preference (45\% had a right preference and 5\% had a left preference). These proportions are similar to those reported by Michel et al. (2014) for a different set of data. Thus, depending upon the task that is used to assess the infant's hand-use preference, there will be different latent classes identified by their developmental trajectories. These differences mark important difficulties for relating the development of infant hand-use preferences to the development of other neurocognitive and sensorimotor functions.

Comparing the infant's membership in the latent class derived from the analysis of their trajectories with their monthly classification by the different decision criteria for the two preference assessment tasks, also revealed important differences. The "Michel" task, with the binomial decision criterion, provides the most reliable prediction of the infant's latent class membership for each of the seven ages. In contrast, the "Fagard" task, with its proportion decision criterion, is much less reliable in its prediction of latent class membership derived from the data obtained from the "Michel" task. Since the "Fagard" task revealed only two latent classes despite identifying three classes of infants at each age, it was not possible to assess the reliability between membership in the "Fagard" latent classes and membership in the "Fagard" monthly assessments. Thus, the "Michel" task is better at predicting latent class membership (derived from the trajectory analysis of data on hand-use preferences from all seven ages) at each age than is the "Fagard" task.

Clearly, the "Michel" and "Fagard" tasks for assessing infant hand-use preferences yield distinct differences in the distribution of preferences for each age period and for the characterization of the latent classes in the trajectory of the development of hand-use preferences. Nevertheless, in this study, both tasks reveal rather high proportions of infants at each month whose hand-use differences are likely to occur by chance. With such high proportions of infants without statistically reliable hand-use preference at each month, it is not surprising that many studies report that infants vary their apparent hand-use preferences for reaching for and manipulating objects during infancy and that those hand-use preferences appear to be unstable. Indeed, our results suggest that studies that examine a single age are likely to report that most infants do not appear to exhibit a statistically reliable hand-use preference at that age. Therefore, tracking the relation between the development of hand-use preferences and the development of other psychological functions (e.g., gestural communication, language skills, and tool-using skills) will require detailed longitudinal investigation of large samples of infants, assessed many times during their development.

The Dubois et al. (2009) quote in our introduction reflects the consensus that it is difficult to identify a stable hand-use preference during infancy. This paper demonstrates that reliable hand-use preferences can be identified during infancy but that half of infants are still developing their preference. Because infant hand-use preference has been examined using an assortment of handedness assessment techniques and different criteria for classifying preference, the stability of infant hand-use preferences only appear to be nonexistent. Our results suggest that the instability is likely a result of the inconsistency in the methods that have been used to assess and categorize infant handedness. Therefore, we strongly recommend that infant hand-use preferences be assessed using a very large number of 
presentations (at least 25) for which there are distinct differences in the hand-used for acquiring an object (no bimanual acquisitions). We also recommend that the infant's preference for acquiring objects be assessed several times (not just two or three) during the six to 14 month age period in order to increase confidence in the estimate of the infant's hand-use preference (cf., Ferre et al., 2010). If neither of these recommendations is followed by those seeking a quick estimate of the infant's hand-use preference for examining its relation to other functions, then research examining the relation of the development of handedness to that of other neuropsychological differences in functioning will continue to reveal contradictory results.

A laterality index of infant hand-use preference exhibits a continuous distribution across individuals with a "right shift" skew similar to that observed in adult handedness (Annett, 1972). However, for neither adults nor infants is there common agreement about how to classify individuals into relatively discrete handedness groups. Using a statistical technique (e.g., binomial test or z-score transformation of the laterality index) for estimating the reliability of a classification ought to be the primary basis for reaching a common agreement for classification for infants. Moreover, since hand-use preferences are developing during infancy, trajectory models ought to be employed for comparing the type of pattern (linear vs. non-linear), direction of change, and rate of change (accelerating vs. decelerating vs. static) of individual and group developmental trajectories (Michel, 2001; Michel et al. 2014). Since critical features of developmental trajectories can be distorted depending on the frequency and intervals of data collection (Adolph, Robinson, Young, \& Gill-Alvarez, 2008; Ferre et al., 2010), the ages for data collection ought to be logically and empirically justified. Of course, many of the issues raised about the study of the development of hand-use preferences during infancy can be applied to the study of the development of hand-use preferences throughout development.

Handedness is an intriguing characteristic of humans because although the right preference predominates across all cultures (and throughout historical and archeological records (e.g., Frost, 1980; Spennemann, 1984; Toth, 1985), there are always left-handers (1 to $18 \%$ of the population depending on the culture and the measure of handedness, e.g., Annett, 2002; McManus, 2002). Since each of the cerebral hemispheres of the human brain control different functions, handedness is both an example of such hemispheric functional asymmetry and it is associated with many other hemisphere-lateralized psychological functions (c.f., Bishop, 2013; Corballis, 2014). Indeed, many left-handers exhibit hemispheric asymmetry of functions that is different from that commonly observed for right-handers (Knecht et al., 2000; Willems, Hagoort \& Casasantos, 2010). Thus, characterizing the development of handedness ought to be an important domain of investigation. We hope that this study prompts more careful investigation of that development during the period of infancy (and childhood, in general).

\section{Acknowledgments}

The data obtained in this article was part of several grant applications by G. F. Michel which were declined review by the Motor Function, Speech and Rehabilitation section of the NIH and which were rated "Not Competitive" by the Development and Learning Sciences section of the NSF. It was supported by G. F. Michel's personal funds. This work was supported in part by a predoctoral fellowship provided by the National Institute of Child Health and 
Human Development (T32-HD07376) through the Center for Developmental Science, University of North Carolina at Chapel Hill, to Julie Campbell. We would like to acknowledge the infants and parents who participated in our longitudinal study, whose patience and help made this research possible. Also, we thank the many graduate and undergraduate students who helped with the coding of infant data: Richard Jordan and Justin Varholick.

\section{References}

Adolph KE, Robinson SR, Young JW, Gill-Alvarez F. What is the shape of developmental change? Psychological review. 2008; 115(3):527. [PubMed: 18729590]

Annett, M. Handedness and brain asymmetry: The right shift theory. Hove, UK: Psychology Press; 2002.

Annett M. The distribution of manual asymmetry. British Journal of Psychology. 1972; 63(3):343358. [PubMed: 4199291]

Babik I, Michel GF. Development of role-differentiated bimanual manipulation in infancy. Child Development. submitted.

Babik I, Campbell JM, Michel GF. Postural influences on the development of infant lateralized and symmetric hand-use. Child Development. 2014; 85:294-307. [PubMed: 23638971]

Baker, DM. Unpublished Master's thesis. DePaul University: Psychology Department; 1998. The use of tree-based methods in the assessment of infant hand preference.

Campbell JM, Babik I, Michel GF. Development of hand-use preferences for unimanual manipulation from 6 to 14 months. Child Development. submitted.

Corballis MC. The evolution and genetics of cerebral asymmetry. Philosophical Transactions of the Royal Society B: Biological Sciences. 2009; 364(1519):867-879.

Corbetta D, Thelen E. Behavioral fluctuations and the development of manual asymmetries in infancy: contributions of the dynamic systems approach. Handbook of Neuropsychology. 2002; 8(1):311330.

Corbetta D, Thelen E. Lateral biases and fluctuations in infants' spontaneous arm movements and reaching. Developmental Psychobiology. 1999; 34(4):237-255. [PubMed: 10331149]

Dubois J, Hertz-Pannier L, Cachia A, Mangin JF, Le Bihan D, Dehaene-Lambertz G. Structural asymmetries in the infant language and sensori-motor networks. Cerebral Cortex. 2009; 19(2): 414-423. [PubMed: 18562332]

Esseily R, Jacquet AY, Fagard J. Handedness for grasping objects and pointing and the development of language in 14-month-old infants. Laterality: Asymmetries of Body, Brain and Cognition. 2011; 16(5):565-585.

Fagard J. The emergence of bimanual coordination during the first year of life. Psychobiology of the Hand. 1998; (147):123.

Fagard J, Lockman JJ. The effect of task constraints on infants'(bi) manual strategy for grasping and exploring objects. Infant Behavior and Development. 2005; 28(3):305-315.

Fagard J, Marks A. Unimanual and bimanual tasks and the assessment of handedness in toddlers. Developmental Science. 2000; 3(2):137-147.

Fennell EB, Satz P, Morris R. The development of handedness and dichotic ear listening asymmetries in relation to school achievement: A longitudinal study. Journal of Experimental Child Psychology. 1983; 35(2):248-262. [PubMed: 6842130]

Ferre CL, Babik I, Michel GF. Development of infant prehension handedness: A longitudinal analysis during the 6-to 14-month age period. Infant Behavior and Development. 2010; 33(4):492-502. [PubMed: 20619463]

Frost GT. Tool behavior and the origins of laterality. Journal of Human Evolution. 1980; 9:447-459.

Gesell A, Ames LB. The development of handedness. The Pedagogical Seminary and Journal of Genetic Psychology. 1947; 70(2):155-175.

Hinojosa T, Sheu CF, Michel GF. Infant hand-use preferences for grasping objects contributes to the development of a hand-use preference for manipulating objects. Developmental Psychobiology. 2003; 43(4):328-334. [PubMed: 15027416]

Ingram D. Motor asymmetries in young children. Neuropsychologia. 1975; 13(1):95-102. [PubMed: 1109467] 
Jacquet AY, Esseily R, Rider D, Fagard J. Handedness for grasping objects and declarative pointing: A longitudinal study. Developmental psychobiology. 2012; 54(1):36-46. [PubMed: 21656764]

Jones BL, Nagin DS, Roeder K. A SAS procedure based on mixture models for estimating developmental trajectories. Sociological Methods \& Research. 2001; 29(3):374-393.

Knecht S, Dräger B, Deppe M, Bobe L, Lohmann H, Flöel A, Henningsen H. Handedness and hemispheric language dominance in healthy humans. Brain. 2000; 123(12):2512-2518. [PubMed: 11099452]

McCormick CM, Maurer DM. Unimanual hand preferences in 6-month-olds: Consistency and relation to familial-handedness. Infant Behavior and Development. 1988; 11(1):21-29.

McManus, C. Right hand, left hand: The origins of asymmetry in brains, bodies, atoms, and cultures. Cambridge, MA: Harvard University Press; 2002.

Michel, GF. Development of infant handedness. In: Lewkowicz, DJ.; Lickliter, R., editors. Conceptions of development: Lessons from the laboratory. New York: Psychology Press; 2002. p. 165-186.

Michel GF. Growth curve analyses are best suited to examine the relation between developmental pathways and selective breeding. Developmental Psychobiolgy. 2001; 39(4):247-250.

Michel, GF. Development of infant manual skills: Motor programs, schemata, or dynamic systems. In: Fagard, J.; Wolff, PH., editors. The development of timing control and temporal organization in coordinated action. NY: Elsevier; 1991. p. 175-199.

Michel GF, Babik I, Sheu CF, Campbell JM. Latent Classes in the Developmental Trajectories of Infant Handedness. 2013

Michel GF, Harkins DA. Postural and lateral asymmetries in the ontogeny of handedness during infancy. Developmental Psychobiology. 1986; 19(3):247-258. [PubMed: 3709979]

Michel GF, Ovrut MR, Harkins DA. Hand-use preference for reaching and object manipulation in 6through 13-month-old infants. Genetic, Social, and General Psychology Monographs. 1985; 111:407-427.

Michel GF, Sheu CF, Brumley MR. Evidence of a right-shift factor affecting infant hand-use preferences from 7 to 11 months of age as revealed by latent class analysis. Developmental Psychobiology. 2002; 40(1):1-13. [PubMed: 11835146]

Michel GF, Tyler AN, Ferre C, Sheu CF. The manifestation of infant hand-use preferences when reaching for objects during the seven-to thirteen-month age period. Developmental psychobiology. 2006; 48(6):436-443. [PubMed: 16886180]

Nelson EL, Campbell JM, Michel GF. Unimanual to bimanual: Tracking the development of handedness from 6 to 24 months. Infant Behavior and Development. 2013; 36(2):181-188. [PubMed: 23454419]

Piek JP, Gasson N, Barrett N, Case I. Limb and gender differences in the development of coordination in early infancy. Human movement science. 2002; 21(5):621-639. [PubMed: 12620715]

Ramsay DS. Onset of unimanual handedness in infants. Infant Behavior and Development. 1980; 3:377-385.

Rat-Fischer L, O'Regan JK, Fagard J. The emergence of tool use during the second year of life. Journal of experimental child psychology. 2012; 113(3):440-446. [PubMed: 22789968]

Rönnqvist L, Domellöf E. Quantitative assessment of right and left reaching movements in infants: A longitudinal study from 6 to 36 months. Developmental psychobiology. 2006; 48(6):444-459. [PubMed: 16886181]

Sacco S, Moutard ML, Fagard J. Agenesis of the corpus callosum and the establishment of handedness. Developmental psychobiology. 2006; 48(6):472-481. [PubMed: 16886185]

Schwarz G. Estimating the dimension of a model. The annals of statistics. 1978; 6(2):461-464.

Siegel, S. Nonparametric statistics for the behavioral sciences. New York: McGraw-Hill; 1956.

Spennemann DR. Handedness data on the human neolithic. Neuropsychologia. 1984; 22:613-615. [PubMed: 6504301]

Thelen E. Motor development: A new synthesis. American psychologist. 1995; 50(2):79. [PubMed: 7879990] 
Thelen E, Corbetta D, Spencer JP. Development of reaching during the first year: role of movement speed. Journal of Experimental Psychology: Human Perception and Performance. 1996; 22(5): 1059. [PubMed: 8865616]

Toth N. Archeological evidence for preferential right-handedness in lower and middle Pleistocene and its possible implications. Journal of Human Evolution. 1985; 14:35-40.

Willems RM, Hagoort P, Casasanto D. Body-specific representations of action verbs: Neural evidence from right- and left-handers. Psychological Science. 2010; 21:67-74. [PubMed: 20424025]

\section{Appendix I}

A. Items used for the 32 presentation Michel Task (the xylophone is presented twice).

A.

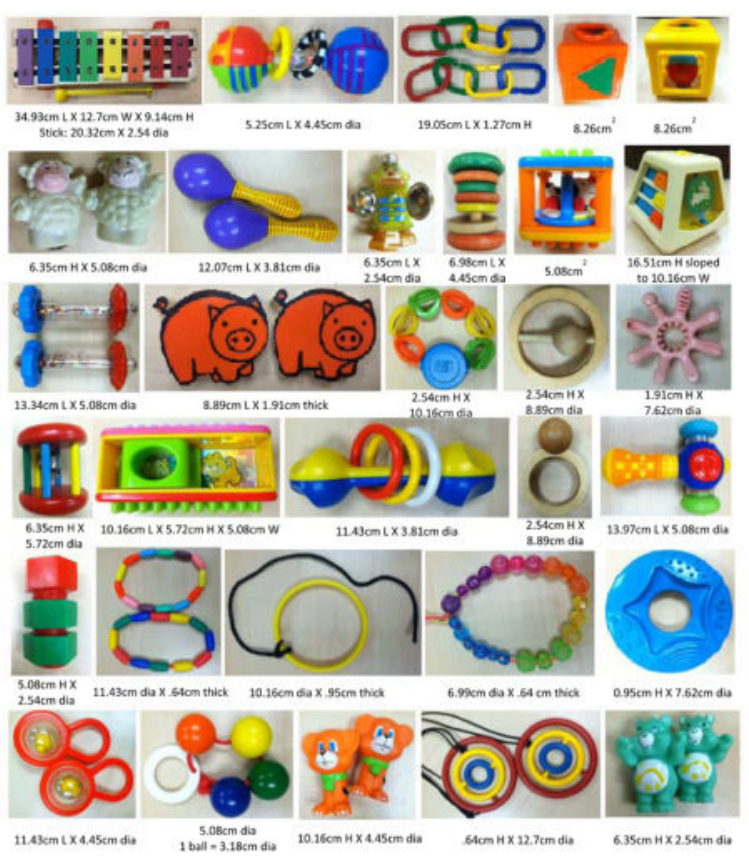

B. Items used for the nine presentation Fagard task (one child figure and the wooden container/tube are presented twice) 
B.

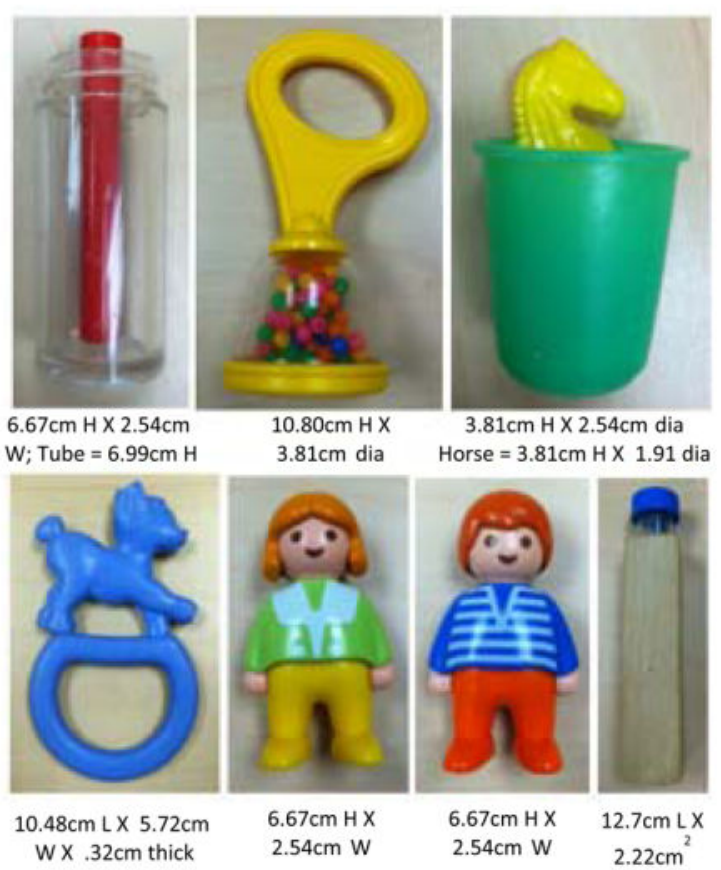

\section{Appendix II}

A. Chi-Square Analyses for the Number of Infants in Each Hand-Use Preference Category for the Fagard Task (Nine Presentations) at Each Month Comparing the Binomial and the Proportion Decision Criteria (Comparing Figure 1A to 1B)

\begin{tabular}{lccccccc}
\hline Infant's Age in Months & $\mathbf{8}$ & $\mathbf{9}$ & $\mathbf{1 0}$ & $\mathbf{1 1}$ & $\mathbf{1 2}$ & $\mathbf{1 3}$ & $\mathbf{1 4}$ \\
\hline$p$ & $0.00^{*}$ & $0.00^{*}$ & $0.00^{*}$ & $0.00^{*}$ & $0.00^{*}$ & $0.00^{*}$ & $0.00^{*}$ \\
$\mathrm{n}$ & 281 & 283 & 287 & 292 & 291 & 289 & 279 \\
$\mathrm{df}$ & 2 & 2 & 2 & 2 & 2 & 2 & 2 \\
Pearson Chi-Square & 57.37 & 49.15 & 39.78 & 35.20 & 42.49 & 36.40 & 30.24 \\
\hline
\end{tabular}

Note.

* Significant at the corrected Bonferroni multiple comparisons level of .007.

B. Chi-Square Analyses for the Number of Infants in Each Hand-Use Preference Category for Michel Task (32 Presentations) at Each Month Comparing the Binomial and the Proportion Decision Criteria (Comparing Figure 2A to 2B)

\begin{tabular}{lccccccc}
\hline Infant's Age in Months & $\mathbf{8}$ & $\mathbf{9}$ & $\mathbf{1 0}$ & $\mathbf{1 1}$ & $\mathbf{1 2}$ & $\mathbf{1 3}$ & $\mathbf{1 4}$ \\
\hline$p$ & .127 & .107 & .131 & .110 & .053 & .026 & $.004^{*}$
\end{tabular}




\begin{tabular}{lccccccc}
\hline Infant's Age in Months & $\mathbf{8}$ & $\mathbf{9}$ & $\mathbf{1 0}$ & $\mathbf{1 1}$ & $\mathbf{1 2}$ & $\mathbf{1 3}$ & $\mathbf{1 4}$ \\
\hline $\mathrm{n}$ & 287 & 285 & 293 & 295 & 293 & 295 & 287 \\
$\mathrm{df}$ & 2 & 2 & 2 & 2 & 2 & 2 & 2 \\
Pearson Chi-Square & 4.13 & 4.46 & 4.06 & 4.42 & 5.87 & 7.27 & 11.30 \\
\hline
\end{tabular}

Note.

* Significant at the corrected Bonferroni multiple comparisons level of .007.

C. Chi-Square Analyses for the Number of Infants in Each Hand-Use Preference Category for the Fagard and Michel Tasks at Each Month Using the Proportion Decision Criterion (Comparing Figure 3A to 3B)

\begin{tabular}{lccccccc}
\hline Infant's Age in Months & $\mathbf{8}$ & $\mathbf{9}$ & $\mathbf{1 0}$ & $\mathbf{1 1}$ & $\mathbf{1 2}$ & $\mathbf{1 3}$ & $\mathbf{1 4}$ \\
\hline$p$ & $.002^{*}$ & $.003^{*}$ & $.004^{*}$ & .147 & $.002^{*}$ & $.001^{*}$ & $.000^{*}$ \\
Total n & 289 & 286 & 293 & 295 & 293 & 295 & 287 \\
$\mathrm{df}$ & 2 & 2 & 2 & 2 & 2 & 2 & 2 \\
Pearson Chi-Square & 12.70 & 11.47 & 11.18 & 3.83 & 12.97 & 13.05 & 31.34 \\
\hline
\end{tabular}

Note.

Significant at the corrected Bonferroni multiple comparisons level of .007.

D. Chi-Square Analyses for the Number of Infants in Each Hand-Use Preference Category for the Fagard Task with the Proportion Decision Criterion and the Michel Task with the Binomial Decision Criterion (Neither Task Includes Bimanual Acquisitions) at Each Month (Comparing Figure 4A to 4B)

\begin{tabular}{lccccccc}
\hline Infant's Age in Months & $\mathbf{8}$ & $\mathbf{9}$ & $\mathbf{1 0}$ & $\mathbf{1 1}$ & $\mathbf{1 2}$ & $\mathbf{1 3}$ & $\mathbf{1 4}$ \\
\hline$p$ & .421 & .199 & .424 & .459 & .929 & .327 & .279 \\
Total n & 278 & 282 & 286 & 292 & 292 & 290 & 280 \\
df & 2 & 2 & 2 & 2 & 2 & 2 & 2 \\
Pearson Chi-Square & 1.73 & 3.23 & 1.72 & 1.56 & .148 & 2.23 & 2.55 \\
\hline Note. & \\
$*$ & \\
Significant at the corrected Bonferroni multiple comparisons level of .007.
\end{tabular}

E. Chi-Square Analyses for the Number of Infants in Each Hand-Use Preference Category for the Fagard Task (with the Inclusion of Bimanual Actions in the Proportion Decision Criterion) and the Michel Task (without the Inclusion of Bimanual Actions in the Binomial Decision Criterion) at Each Month (Comparing Figure $4 \mathrm{~A}$ to Figure 1B)

\begin{tabular}{lccccccc}
\hline Infant's Age in Months & $\mathbf{8}$ & $\mathbf{9}$ & $\mathbf{1 0}$ & $\mathbf{1 1}$ & $\mathbf{1 2}$ & $\mathbf{1 3}$ & $\mathbf{1 4}$ \\
\hline$p$ & $.000^{*}$ & $.000^{*}$ & $.000^{*}$ & $.000^{*}$ & $.000^{*}$ & $.000^{*}$ & $.000^{*}$ \\
Total n & 279 & 283 & 287 & 292 & 292 & 290 & 280
\end{tabular}




\begin{tabular}{lccccccc}
\hline Infant's Age in Months & $\mathbf{8}$ & $\mathbf{9}$ & $\mathbf{1 0}$ & $\mathbf{1 1}$ & $\mathbf{1 2}$ & $\mathbf{1 3}$ & $\mathbf{1 4}$ \\
\hline $\mathrm{df}$ & 2 & 2 & 2 & 2 & 2 & 2 & 2 \\
Pearson Chi-Square & 53.92 & 67.63 & 55.40 & 48.89 & 44.30 & 34.31 & 28.42 \\
\hline
\end{tabular}

Note.

* Significant at the corrected Bonferroni multiple comparisons level of .007. 


\section{Highlights}

- Infant hand preference was assessed with 2 tasks and 2 criteria for classification

- The 2 tasks and criteria produced differences in infant handedness classification

- Latent class analyses of developmental trajectories of handedness were different

- Differing tasks can create confusion about the stability of infant hand preference 


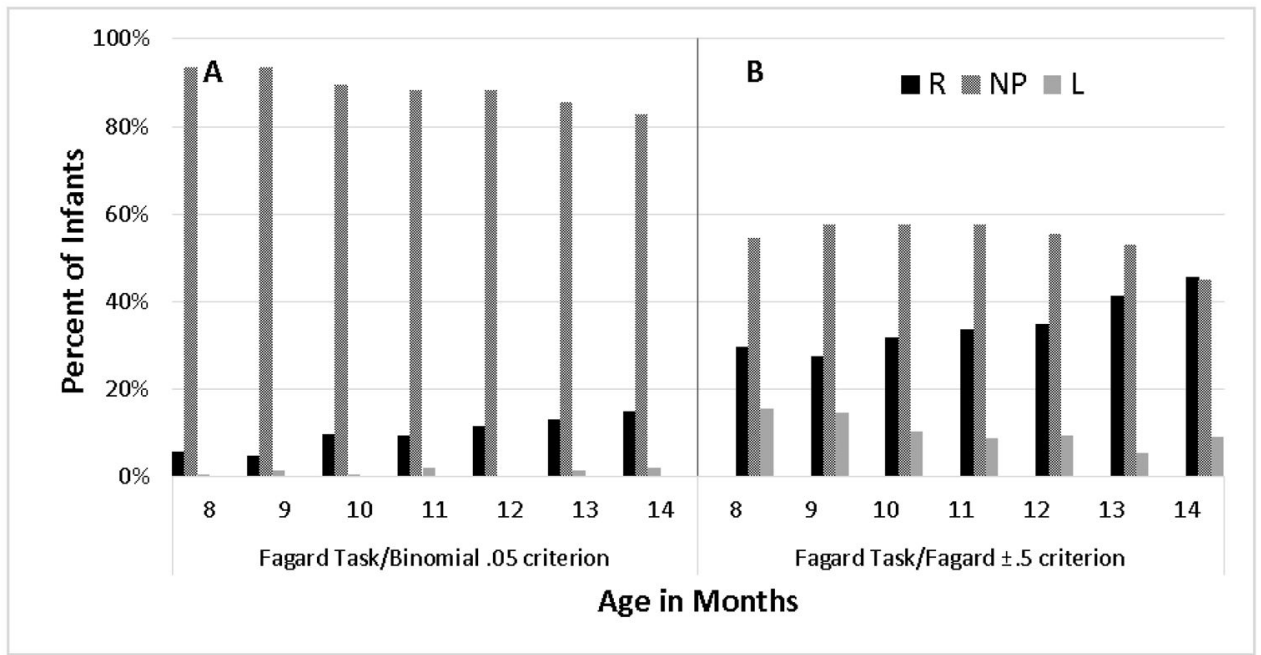

Figure 1.

Percent of Infants in Each Handedness Category for 8-Item Procedure at each Month. 


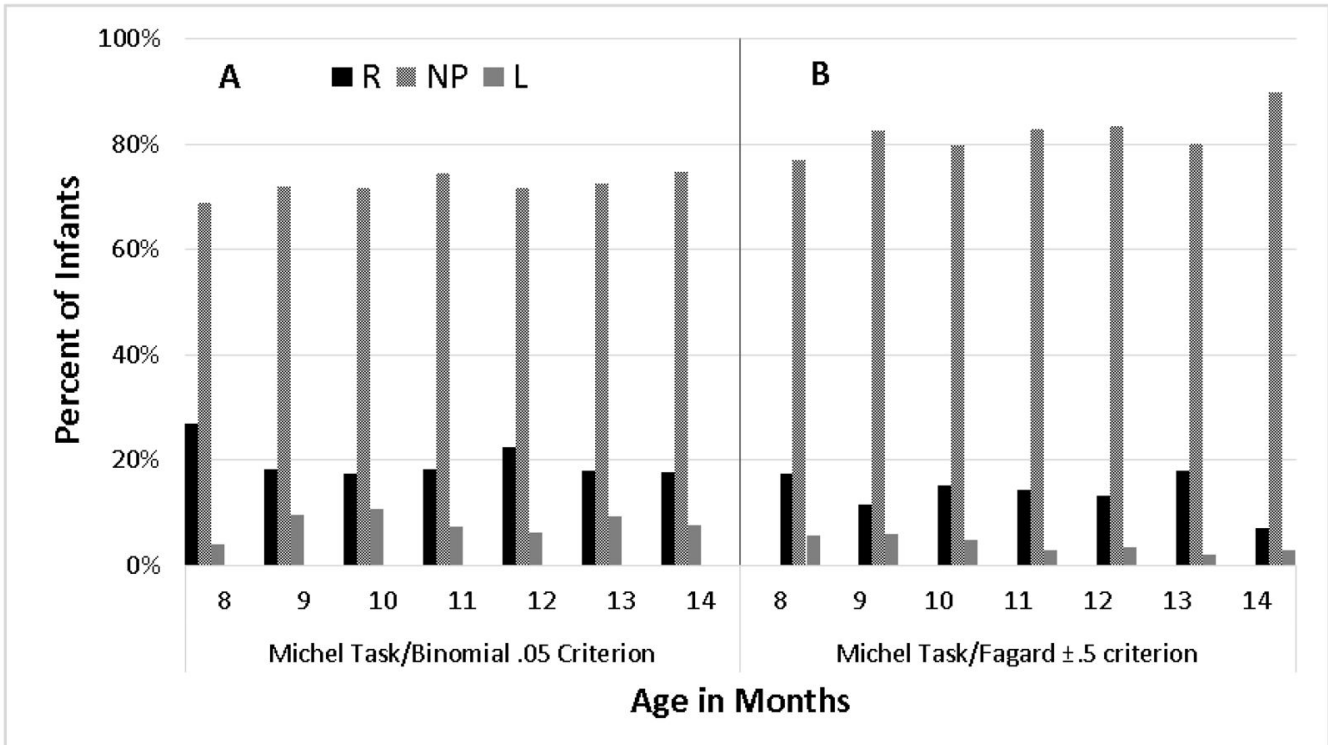

Figure 2.

Percent of Infants in Each Handedness Category for 32-Item Procedure at each Month. 


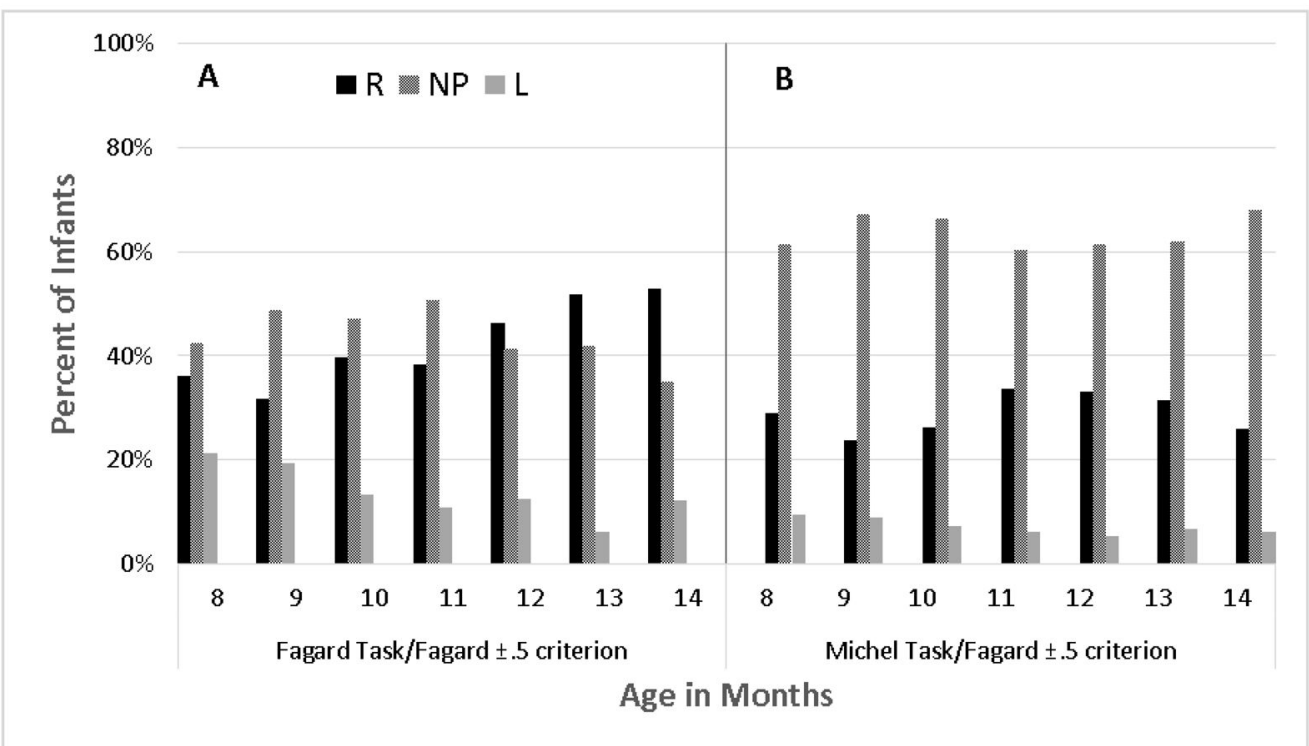

Figure 3.

Percent of Infants in Each Handedness Category for 32-Item and 8-Item Procedure at each Month. 


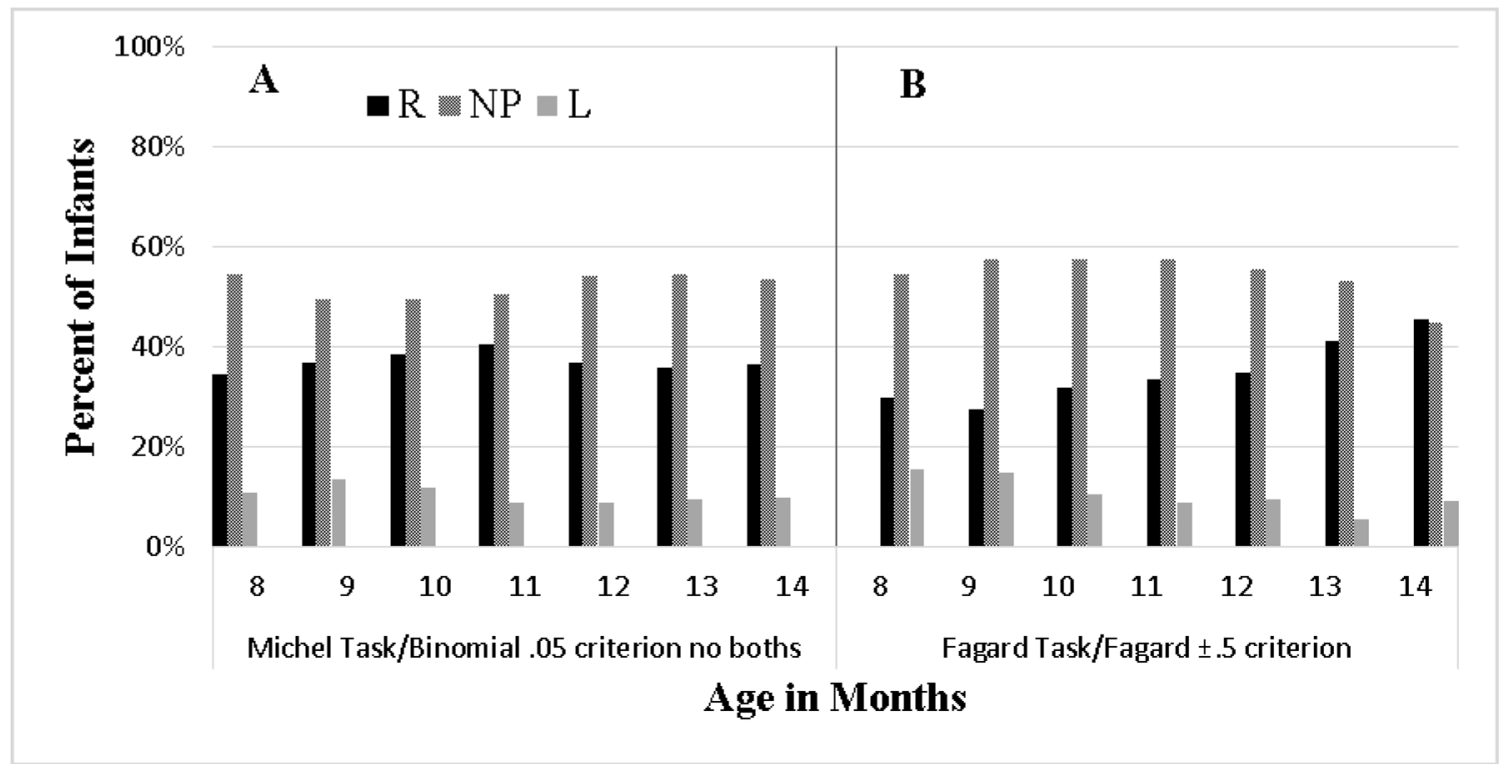

Figure 4.

Percent of Infants in Each Handedness Category for 32-Item and 8-Item Procedure at each Month. 


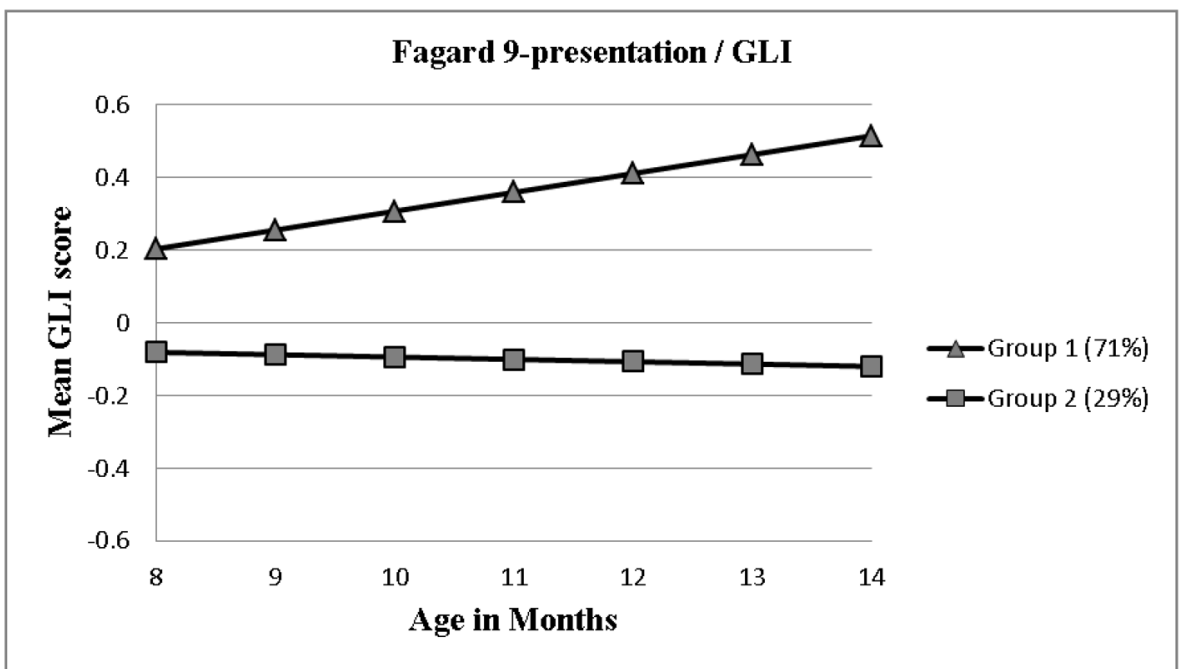

Figure 5.

Trajectories of the two latent groups identified via the "Fagard" nine presentation method. 


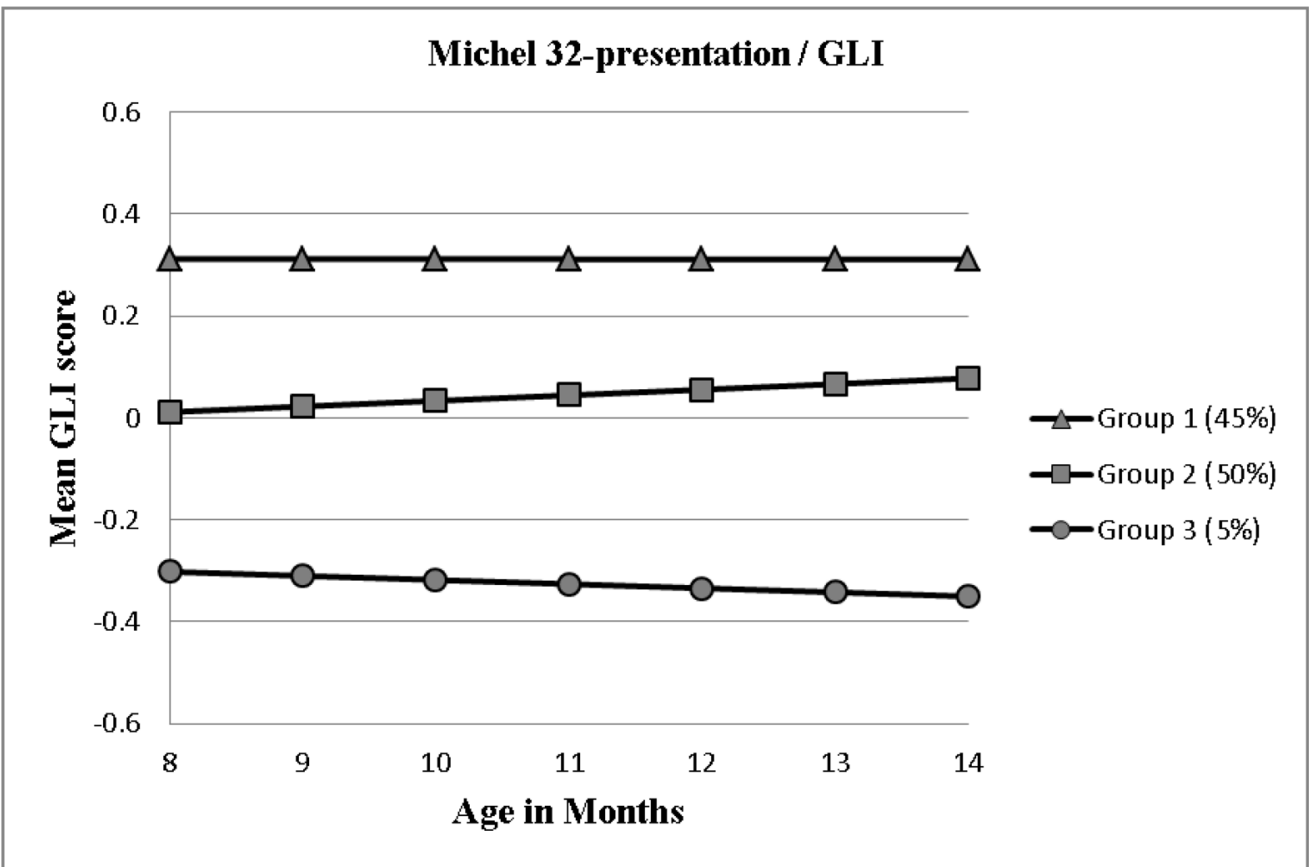

Figure 6.

Trajectories of the three latent groups identified via the "Michel" 32 presentation method. 
Table 1

Percentage of Infants in each Handedness Group at 14 months for the Current Study as Compared to the Esseily et al., 2011 Study

\begin{tabular}{lccc}
\hline & Right-handed & No Preference & Left-handed \\
\hline Esseily et al. (2011) $\mathrm{n}=22$ & 63 & 23 & 14 \\
Current Study, $\mathrm{n}=140$ & 46 & 45 & 9 \\
\hline
\end{tabular}




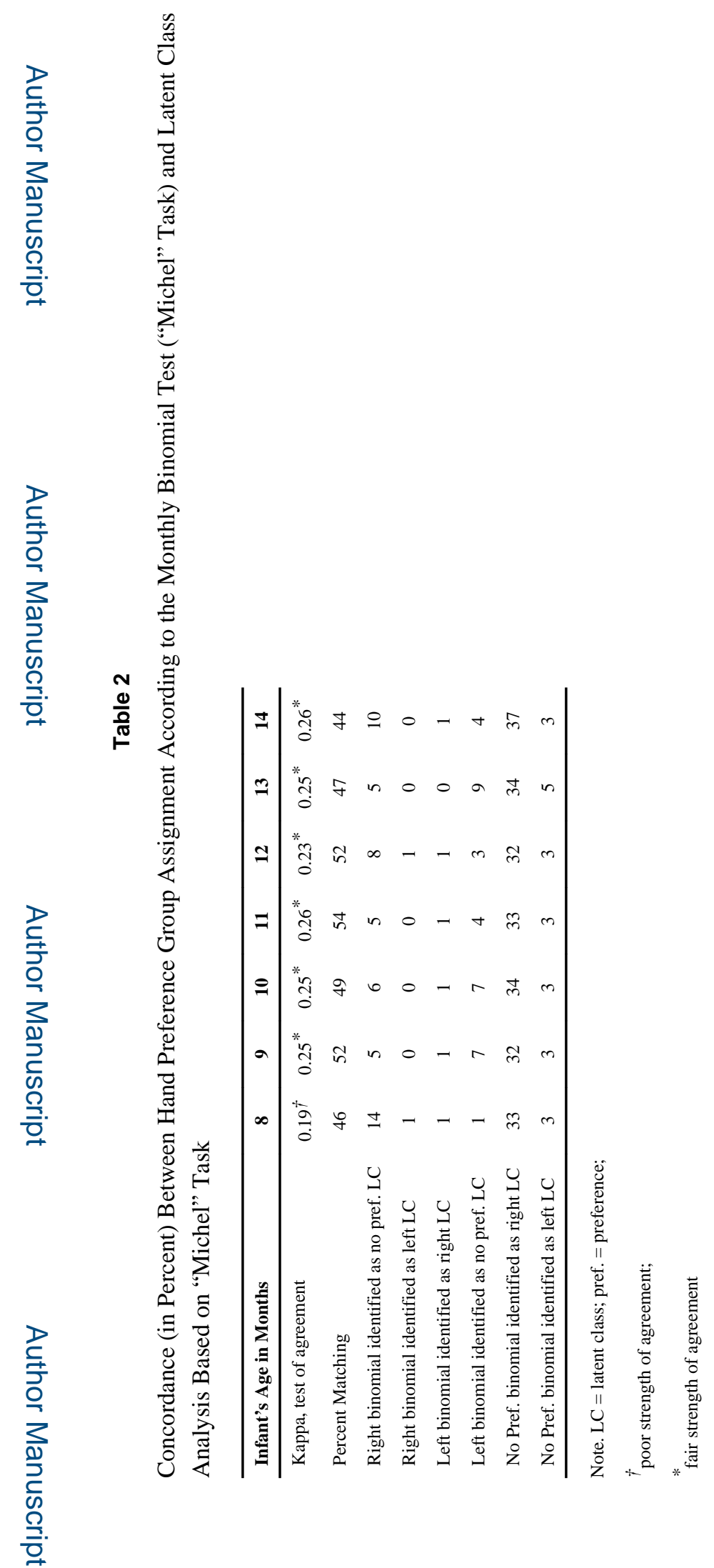

Infant Behav Dev. Author manuscript; available in PMC 2016 May 01. 


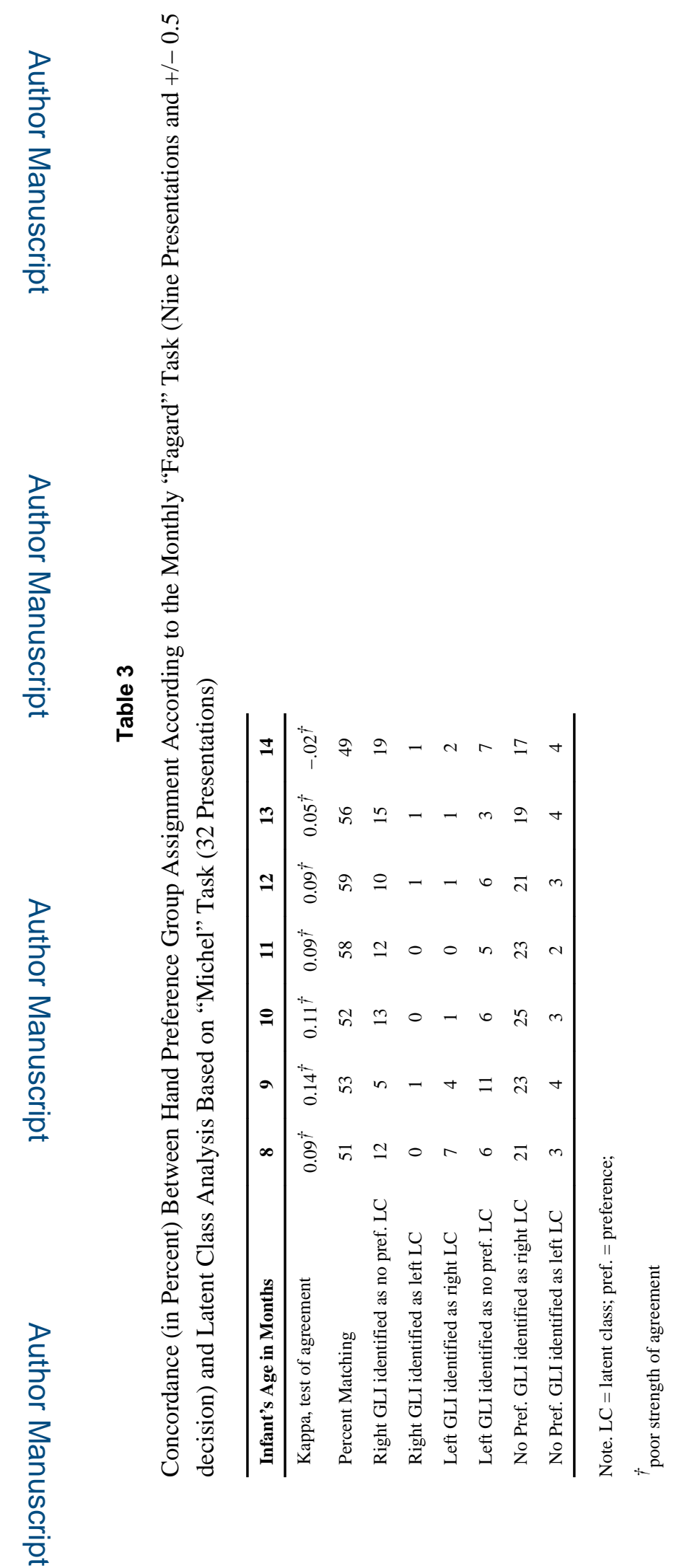

Infant Behav Dev. Author manuscript; available in PMC 2016 May 01. 